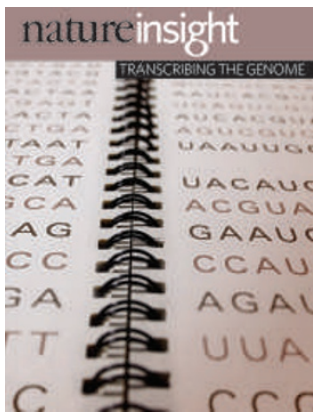

Cover illustration Artwork by N. Spencer

\section{Editor, Nature}

Philip Campbell

Publishing

Nick Campbell

Claudia Deasy

Insights Editor

Lesley Anson

\section{Production Editor}

Davina Dadley-Moore

Senior Art Editor

Martin Harrison

Art Editor

Nik Spencer

Sponsorship

Amélie Pequignot

Reya Silao

Production

Jocelyn Hilton

Marketing

Elena Woodstock

Emily Elkins

Editorial Assistant

Emma Gibson

\title{
TRANSCRIBING THE GENOME
}

$\mathrm{R}$ ecent technological advances are transforming our understanding of how the DNA sequence of the genome is transcribed into its functional output of RNA and protein. Researchers are uncovering new layers of complexity on many levels, ranging from the mechanism by which genes are transcribed into RNA to how genetic changes can give rise to disease.

Advances in the detailed mapping of transcription factors across the genome are revealing, for example, unexpected rate-limiting steps during the initiation of gene transcription by RNA polymerase. And such analyses are defining the regulatory regions of genes, as well as the factors that bind to these regions. The distribution of nucleosomes along DNA can now also be finely mapped, showing the dynamic interplay between the packaging of DNA into chromatin and the binding of transcription factors to regulatory regions.

Improved techniques for following the threedimensional interactions of chromosomes in the nucleus are allowing the effect of such interactions on gene activation and silencing to be explored and are offering glimpses of the poorly understood substructure of the nucleus. Furthermore, highthroughput sequencing methods are uncovering different classes of RNA that are transcribed from various regions of the genome but not translated into proteins, raising the question of the functional importance of these RNAs.

Complementing such experimental methods, computational approaches are revealing the importance of mutations that promote susceptibility to disease not by directly affecting protein-coding sequences but instead by disrupting gene regulatory regions. And computational network approaches are aiding our understanding of the systems-level changes driven by disease-associated mutations.

These Reviews are intended to convey some of the current excitement in the transcription and genomics fields, and we are grateful to the authors and reviewers for their contributions.

Alex Eccleston and Magdalena Skipper, Senior Editors

\section{REVIEWS}

186 Defining mechanisms that regulate RNA polymerase II transcription in vivo

N. J. Fuda, M. Behfar Ardehali \& J. T. Lis

193 The logic of chromatin architecture and remodelling at promoters
B. R. Cairns

199 Genomic views of distant-acting enhancers

A. Visel, E. M. Rubin \&

L. A. Pennacchio

206 Implications of chimaeric non-co-linear transcripts

T. R. Gingeras

212 Chromosome crosstalk in three dimensions
A. Göndör \& R. Ohlsson

218 Molecular networks as sensors and drivers of common human diseases

E. E. Schadt

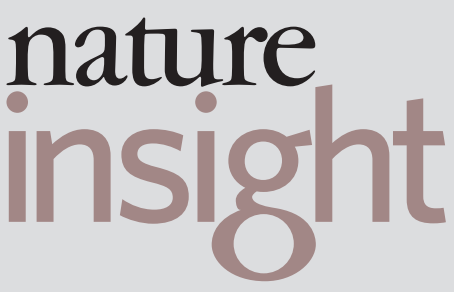

